\title{
'Dam it - it's easy!' - or is it?
}

\author{
K. Marshall
}

\section{In brief}

Dentists the world over seem to still have issues in making rubber dam application a routine part of practice, even in situations where patients are obviously at risk. In this article, the author - who has delivered hundreds of seminars and hands on tuition in a quick and easy method of dam application (the 'Dam it - it's Easy!' series) to dentists in the UK, Europe, North America and Asia - highlights the similarity of the misconceptions existing in regard to its use by dentists the world over. The valuable advantages to the environment in the dental treatment room by dental dam application are considered underused. The protection offered by routine rubber dam application is second to none in situations that are potentially physically hazardous to patients.

A BDJ letter published in February 2017 entitled 'Endodontics: No rubber dam, no root canal $^{1}$ made me think. It is certainly concerning and worthy of comment that dentists electively choose to ignore the evidenced benefits of rubber dam during various modalities of treatment.

The British Medical Journal report ${ }^{2}$ alluded to by Dr Julian Webber in that BDJ letter, despite its long title and fairly complex nature, clearly confirms this poor uptake in the US cohort of dentists studied. There is the suggested caveat that perhaps the participants were not entirely representative of 'dentists at large' as they had an interest in clinical research which was evidenced by joining this group rather than being recruited randomly (is this intended to suggest a possible expectation of greater rubber dam use?). However, the group were eventually considered to have much in common with dentists at large and to offer diversity in these characteristics. This said, generally, the findings for rubber dam use by dentists in this study and others over the last thirty years confirm disappointing levels of use

${ }^{1}$ Cardiff University, PGMDE, Plas Canol, Llanybri, Carmarthen, SA33 5HJ

Correspondence to: Keith Marshall

Email: drkeithfm@gmail.com

Refereed Paper. Accepted 10 March 2017

DOI: 10.1038/sj.bdj.2017.489 even in situations that threaten patient safety. ${ }^{3,4}$ Universal and easily applicable guidelines are suggested to determine usage in all situations.

An earlier study ${ }^{5}$ on rubber dam use in the UK based on a cohort of over a thousand dentists showed that attitudes were fairly clear in regard to rubber dam use and these opinions recur time and time again when practitioners are questioned. During hundreds of personal seminars delivered on the topic over the last 30 years, both in the UK and many other countries, the same three answers are prominent when dentists get questioned as to their perceptions of rubber dam. These are always: 1) It's difficult; 2) It's time consuming; and 3) My patients wouldn't like it. The answers are so entirely predictable that I have them confidently pre-loaded in the seminar on the next slide!

Practitioners are generally well aware of the potential for adverse safety events that threaten the patient being treated, and the potential for reduction in treatment efficacy and possibly standards that are related to the non-use of dental dam. The seeming justification for non-use - and this is also supported by the nuanced qualitative comments at the end of the $B M J$ study referenced by Dr Webber - is that in regard to the dentists concerned, they have not had these problems, and rubber dam is perceived as being difficult and not liked by patients. Routine users of dam would not consider either of these reasons viable from a usage or a patient safety point of view. Also, they are not justified from the published evidence available in regard to the high levels of success demonstrated in a well-structured and prize winning study by a young dentist (then a 'VT'/DF1) on a patient cohort that had never experienced dam placement before. ${ }^{6}$ Age range within the cohort was from 15 to 78 years with ten patients over 55 . The ratio of acceptance/preference increased as the study progressed reflecting, it was considered, an improvement in operator skills. There would appear to be few unsuitable patients, and towards the end of the study $84.9 \%$ actually preferred treatment with dam in place! This reflects my own experience entirely, and anecdotally that of other regular users as well.

Another area of concern is the apparent poor knowledge throughout the profession in regard to the effect delivered to the operating room environment by having dam in place when the air-turbine is being used. The airturbine is an effective 'aerosoliser' of all the microbial contaminants in the oral cavity environment (saliva, blood, crevicular fluid, exhaled products from the lungs and alimentary tract, etc). These are then efficiently spread around by the turbine either to remain suspended for the long term or to settle, around an area of sometimes more than two metres from the patient's mouth and repeatedly 
re-contaminate instruments, uniforms, work surfaces and equipment. This is then added to by the microbial load from the next patient. A 'wipe up - swab down' by the dental nurse for a couple of minutes, which is the usual regime, cannot be wholly effective!

With rubber dam in place, $98.5 \%$ of the microbial content of such aerosols is shown to be eliminated, as is continued touch re-contamination of the gloved hands of dentist and nurse with the patient's oral mucosa. Two excellent, almost simultaneous studies either side of the Atlantic in the UK and USA illustrated this remarkable effect of rubber dam on the aerosol with similar findings in each. ${ }^{7,8}$ Workspace contamination is unaffected by the wearing of gloves, masks and other PPE, and rubber dam has been shown to be an effective modifier of this. This latter effect would be expected to serve as a driver not only to greater patient safety but to more effective personal protection for the operator and team, and a front line strategy.

While it could be argued that it is the busy general dental service practice with larger patient throughput that needs such a strategy, with multiple aerosolised contamination from sometimes 40 patients or more in a daylist, the available evidence in regard to nosocomial infections arising in the dental operatory is thin, with there being no surveillance programmes in place to assess this, or even the efficacy of current decontamination programmes in this respect.

The defence organisations and also the General Dental Council in a professional conduct committee (PCC), would, I imagine, no doubt take a dim view of a practitioner electively choosing not to suitably incorporate rubber dam application into the treatment strategy when it was clearly indicated and appropriate to minimise hazard to the patient being treated, and this mindset, perhaps, should be appropriate for the profession as a whole.

1. Webber J. Endodontics: No rubber dam, no root canal. Br Dent J 2017; 222: 142.

2. Gilbert G H, Riley J L, Eleazer P D, Benjamin P L, Funkhouser E; National Dental PBRN Collaborative Group. Discordance between presumed standard of care and actual clinical practice: the example of rubber dam use during root canal treatment in the national dental Practice-Based research network BMJ Open 2015; 5: e009779.

3. Lynch C D, McConnell R J. Attitudes and use of rubber dam by Irish general dental practitioners. Int Endod J 2007; 40: 427-432.

4. Ahmed H M, Cohen H S, Levy G et al. Rubber dam application in endodontic practice: an update on critical educational and ethical dilemmas. Austr Dent J 2014; 59: 457-463.

5. Marshall K, Page J. The use of rubber dam in the UKa survey. Br Dent J 1990; 169: 286-291.

6. Gergely E. Rubber Dam Acceptance. Br Dent J 1989; 167: 249-252.

7. Cochran M A, Miller C H, Sheldrake M A. The efficacy of the rubber dam as a barrier to the spread of micro-organisms during dental treatment. JADA 1989; 199: 141-144.

8. Evans D, Samaranayake L P, Reid J. The use of rubber dam in reducing atmospheric contamination. J Dent Child 1989; 56: 442-444. 